\title{
Preliminary two-point observations of the mid-altitude cusp by Cluster PEACE and FGM
}

\author{
I. Krauklis ${ }^{1}$, A. N. Fazakerley ${ }^{1}$, C. J. Owen ${ }^{1}$, P. J. Carter ${ }^{1}$, M. W. Dunlop ${ }^{2}$, A. J. Coates ${ }^{1}$, S. Szita ${ }^{1}$, \\ M. G. G. T. Taylor ${ }^{1}$, P. Travnicek ${ }^{1}$, G. Watson ${ }^{1}$, and R. J. Wilson ${ }^{1}$ \\ ${ }^{1}$ Mullard Space Science Laboratory, Department of Space and Climate Physics, University College London, UK \\ ${ }^{2}$ Blackett Laboratory, Imperial College of Science Technology and Medicine, London, UK
}

Received: 14 May 2001 - Revised: 16 July 2001 - Accepted: 3 September 2001

\begin{abstract}
On 1 October 2000, Cluster spacecraft Samba (Cluster 3) and Tango (Cluster 4) made an outbound crossing of the northern mid-altitude $\left(4.7 R_{E}\right)$ cusp region, moving roughly parallel to the noon meridian. We present preliminary observations from this interval made by the PEACE and FGM instruments. The interplanetary magnetic field at the magnetopause is estimated to have turned south at the time of our observations, based on ACE data as well as a rough estimate of the time taken for the solar wind to travel between ACE and the magnetopause. Cluster 3 encountered the low-latitude boundary layer (LLBL) between 12:20:30 to 12:26:00 UT, and the cusp region between 12:26:00 and 12:32:30 UT. Cluster 4 encountered the LLBL between 12:22:00 to 12:29:00 UT, and the cusp region between 12:29:00 and 12:38:00 UT. During the interval between the two spacecraft passages through these regions, the open/closed field line boundary was observed to move equatorward by $0.33^{\circ}$ invariant latitude, while the latitudinal extent of the cusp region increased by $0.5^{\circ}$. Both of these observations are consistent with the ongoing reconnection at the sub-solar magnetopause. The magnetic field data indicate that Cluster encountered four field-aligned longitudinally extended current sheets. The most equatorward of these is consistent with the location of a Region 1 current sheet. Two current sheets were observed in the vicinity of the cusp region, though neither of these were thin current sheets. The fourth current sheet was observed in the mantle region and was largely unaffected by the latitudinal expansion of the cusp region.
\end{abstract}

Key words. Magnetospheric physics (current systems; energetic particles, precipitating; magnetopause, cusp and boundary layers)

\section{Introduction}

Reconnection between the interplanetary magnetic field (IMF) and the magnetosphere, first proposed by

Correspondence to: I. Krauklis (ick@mssl.ucl.ac.uk)
Dungey (1961), is the principal mechanism whereby solar wind mass and energy gain entry to the magnetosphere. Extensive studies of reconnection in the sub-solar magnetopause region during intervals of southward IMF have been made (Reiff et al., 1980; Hones, 1984; Smith and Lockwood, 1996). The low and mid-altitude cusp regions have been a favoured region to observe this entry mechanism as reconnected field lines threading a (spatially) large region of the magnetopause are mapped into the much smaller cusp region. The general morphology of the plasma populations seen at a low altitude $(800 \mathrm{~km})$ has been described by Newell and Meng (1988). One of their observations characterized the cusp by an increase in the plasma density and a reduction in plasma temperature. They considered the cusp region to be a spatial structure in which a spacecraft moving to an increasing invariant latitude would first encounter the magnetopause boundary layer and then move into the cusp region. The former consists of plasma whose bulk density and temperature are intermediate between the plasma sheet and the cusp regions. Lockwood and Smith (1994), noting that flux transfer events (FTEs) first observed by Russell and Elphic (1979) are a manifestation of magnetic merging, developed a time-dependent reconnection model in which the reconnection rate varies. This model explains the plasma energy-time dispersion patterns often seen in the cusp region (Woch and Lundin, 1992; Lockwood et al., 1998). Observations of the Birkeland current system in these regions have also been made (Iijima and Potemra, 1976; Erlandson et al., 1988; de la Beaujardiere et al., 1993). Longitudinally-extended field-aligned current sheets can be identified by their effect on the GSM $Y$ component of the magnetic field (Rostoker, 1980). Reversals in the gradient of the GSM $Y$ component of the perturbation magnetic field $\delta B_{\perp Y}$ have been used to identify boundaries between current sheets (de la Beaujardiere et al., 1993). In this paper, a two-point observation is described in which we examine the mid-altitude $\left(4.7 R_{E}\right.$ ) cusp with data from the Cluster PEACE and FGM instruments. A brief description of the instruments is given in the next section followed by an overview of the event. Finally, these observations are 


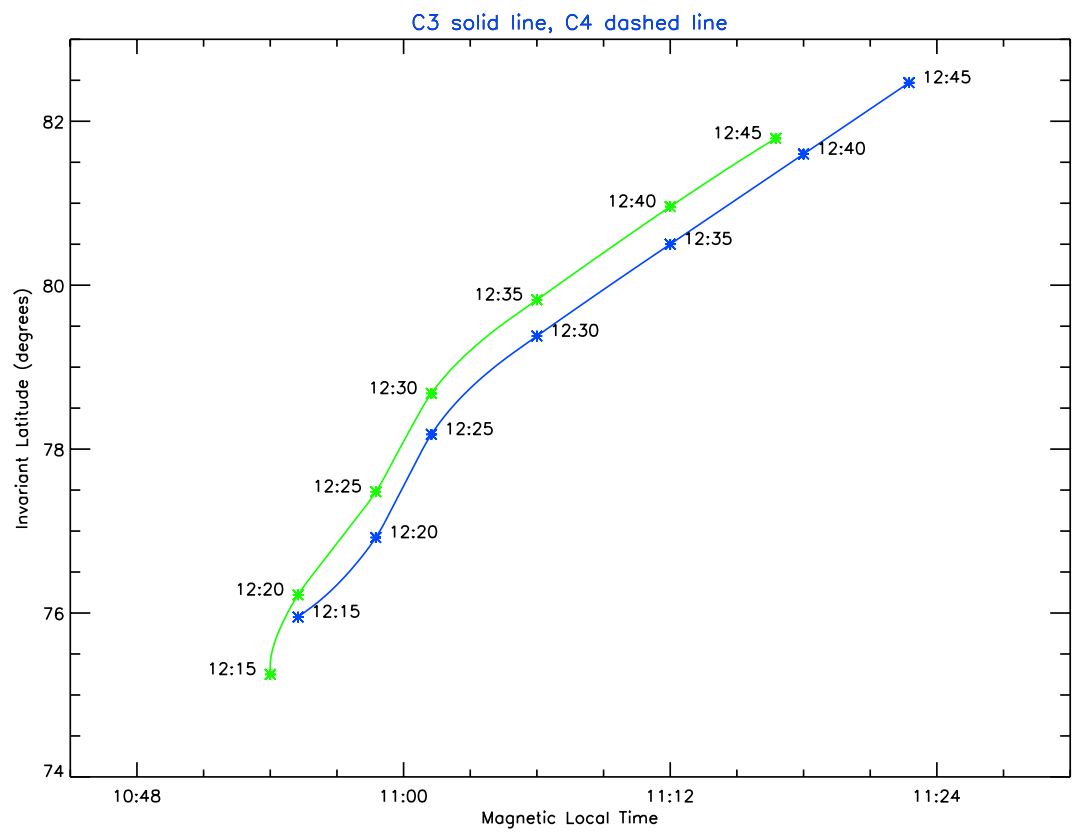

Fig. 1. Spacecraft orbits plotted in MLT against invariant latitude for the interval 12:15 to 12:45 UT; Cluster 3 is the blue line and Cluster 4 is the green line.

discussed in terms of reconnection at the magnetopause.

\section{Instrumentation}

Electron measurements from PEACE (Plasma Electron And Current Experiment) (Johnstone et al., 1997) measurements from FGM (Flux Gate Magnetometer) (Balogh et al., 2001, this issue) consists of two sensors with hemispherical electrostatic analyzers which measure the three-dimensional velocity distribution of electrons between $1 \mathrm{eV}$ and $26 \mathrm{keV}$. Each sensor measures a different overlapping part of the energy range. On 1 October 2000 , the energy range measured by the low energy electron analyzer (LEEA) covered $1 \mathrm{eV}$ to $1 \mathrm{keV}$, and the high energy electron analyzer (HEEA) covered $40 \mathrm{eV}$ to $26 \mathrm{keV}$. The FGM instrument consists of two tri-axial sensors capable of sampling up to 67 vectors/s. In this study, we use spin resolution ( $4 \mathrm{~s})$ magnetic field data.

\section{Overview of event}

On 1 October 2000 (day of year 275), the Cluster spacecraft made an outbound crossing of the northern mid-altitude (approximately $4.7 R_{E}$ ) cusp region. The magnetic local time of the cusp encounter was 11:00 MLT and the orbit was roughly parallel to the noon-midnight meridian. Cluster was in the commissioning phase of the mission, such that only PEACE data from Cluster 3 (Samba) and Cluster 4 (Tango), and FGM data from Cluster 2 (Salsa), Cluster 3 and Cluster 4 were available. For this study, we only use data from Cluster 3 and 4 . These spacecraft were separated by approximately $1300 \mathrm{~km}$. The separation was principally along the orbit path and Cluster 4 lagged behind Cluster 3. Figure 1 shows the spacecraft footprints, with Cluster 3 as the blue line and Cluster 4 as the green line, in MLT against invariant latitude for the time interval 12:15 to 12:45 UT (the discontinuous changes are a facet of the ephemeris data). The difference in Cluster 3's and Cluster 4's location is principally in invariant latitude. In terms of invariant latitude, Cluster 4 lagged behind Cluster 3. The separation, in magnetic local time, between the two spacecraft at any given invariant latitude for the duration shown in Fig. 1 is always less than 4 min. The GSE $B_{Z}$ component of the IMF observed at ACE (PI, N. Ness, access courtesy of CDAweb) turned southward at 11:33 UT. At this time, ACE was $226 R_{E}$ sunward. Using the solar wind velocity observed at ACE (PI, D. J. McComas, access courtesy of CDAweb), the solar wind travel time to the Earth's magnetopause along the Earth-Sun axis is approximately $55 \mathrm{~min}$. However, ACE was also $29 R_{E}$ dawnward and $12 R_{E}$ northward of the Earth-Sun axis. Assuming that, on small scales (namely the distance of ACE from the earthsun axis) the IMF is planar, then this ACE's position will effect the travel time noted above. For instance, at 11:33 UT, the IMF resolutes are approximately $(-2.0,+1.5,0.0)$. This IMF vector will intercept the Earth-Sun axis approximately $22 R_{E}$ earthward from ACE, thereby reducing the travel time by approximately 5 minutes. It should be noted that the IMF is quite variable at this time and this factor should be used as an indication of the uncertainty in the travel time, rather than a correction factor.

An overview of the PEACE data from the interval is given in Fig. 2, which shows six $4 \mathrm{~s}$ resolution energy-time spectrogram panels. The three panels in Fig. 2a correspond to data observed at Cluster 3. Each panel is comprised of data 
collected by both LEEA and HEEA sensors. The spectra are plotted in differential energy fluxm, which is not regarded as highly accurate since it is produced by combining raw data and preliminary calibrations: however, this is sufficient for a qualitative study. The top panels shows observations of electrons travelling along the direction of the magnetic field, i.e. field-aligned, and the middle panel shows electrons moving perpendicular to the magnetic field, while the bottom panel corresponds to field opposed electrons. Figure $2 \mathrm{~b}$ is in the same format as Fig. 2a, but depicts data taken at Cluster 4 . The high values of differential energy flux at energies from $1 \mathrm{eV}$ up to as high as $10 \mathrm{eV}$ are most likely photoelectrons and secondary electrons from the spacecraft. High energy $(>1 \mathrm{keV})$ electrons in the early parts of the interval with higher fluxes perpendicular to the magnetic field are trapped plasma sheet electrons on closed field lines. There is a noticeable change in the characteristics of the observed electron populations around 12:21 UT in Cluster 3 and around 12:22 UT in Cluster 4. For both data sets, the high energy population considerably reduces in flux. At the same time a lower energy $(100 \mathrm{eV})$ population is observed. At 12:26 UT in Cluster 3 and 12:29 UT in Cluster 4, there is a reduction in the flux of the photoelectrons $(<10 \mathrm{eV})$ which is most likely due to the spacecraft being discharged by the arrival of magnetosheath ions. As such, these photoelectron drop outs are good indicators of the cusp (D. Winningham, private communication). The region between the disappearance of the higher energy population and the cusp region has a slightly lower flux and higher mean energy in comparison to the cusp and is consistent with the low-latitude boundary layer (LLBL) (Newell and Meng, 1988). At 12:32:30 UT in Cluster 3 and at 12:38 UT in Cluster 4, both spacecraft exited the cusp region and entered the mantle region characterised by a reduction in flux (Rosenbauer et al., 1975). This transition is characterised by a reduction in the flux of electrons between 10 to $100 \mathrm{eV}$ and an increase in the flux of photoelectrons.

In order to quantify these observations, (bulk) moments of the electron populations observed by the HEEA sensor have been used. HEEA was chosen to avoid including photoelectrons. The entire velocity space volume observed by HEEA is used to calculate the bulk moments. It should be noted that these moments were calculated on board using preliminary calibrations and should be considered as representative, rather than fully accurate. Total scalar temperature and number density moments are shown in the upper and lower panels of Fig. 3, respectively. For both panels, blue colouring relates to the observations made at Cluster 3 and the green colouring relates to the observations at Cluster 4 . The variations in electron density and temperature are consistent with previous observations (Lockwood and Hapgood, 1998) of the magnetosphere which is populated by hotter, rarefied plasma, and the cusp region which is populated by cooler, dense magnetosheath-like plasma. The disappearance of the higher energy population observed in the spectrograms is observable as a rapid drop in the temperature (it should be noted that these moments represent an amalgam of the two distinct physical populations). This disappearance is interpreted as a transition from a region with closed field lines to a region with open field lines, (termed the open-closed field line boundary, OCB). In both data sets, the temperature drop is not discontinuous and hence, there is some uncertainty in the precise location of the OCB. In addition to identifying the OCB by changes in the characteristics of the electron population, other techniques, such as observing broadband electrostatic noise (Franz et al., 1998) and observations of upwelling ionospheric plasma populations (André et al., 1990) validation, are beyond the scope of this paper. The precise location of the OCB is not critical to this study. The only significant fact here is that Cluster 4 observes this temperature transition further equatorward in comparison to Cluster 3 . The vertical dotted lines with the common label 'OCB' mark the approximate position of the OCB. The cusp region is identified from the number density. The rapid increase in the number density, observable in both data sets, occurred at approximately $78.5^{\circ}$ and marks the equatorward edge of the cusp region. It is identified by the two vertical lines and the common label ' $E$ '. The poleward edge of the cusp, identified by the sharp decrease in density, is also identified by two vertical lines and is labelled ' $P$ '.

The magnetospheric boundary region depicted in Fig. 2 is expected to contain a number of field-aligned current systems (e.g. see Fig. 3 in Cowley et al., 1991). We have examined the Cluster magnetic field data for evidence of such currents. Figure 4a shows the three components and magnitude of the observed magnetic field (solid line) and background field (dashed line) in the GSM coordinate system. The background field was computed by smoothing the total field with a 400 sample point running mean boxcar filter. Figure $4 \mathrm{~b}$ depicts data observed at Cluster 4. The magnetic field is primarily in the $X-Z$ GSM plane and perturbations from the background field are primarily in the $Y$ direction (note different scales on the plots). Rostoker (1980) notes that close to 12:00 MLT, a longitudinally extended field-aligned current would cause perturbations in the $Y$ component of the magnetic field. Figure 5 shows the three components of the perpendicular perturbation magnetic field in GSM coordinates for both spacecraft. The bottom panel shows the number density. Blue colouring relates to the observations made at Cluster 3 and the green colouring relates to the observations at Cluster 4. The vertical dotted lines represent the approximate latitude of the OCB. The solid vertical lines represents the current sheet boundaries inferred by changes in gradient in $\delta B_{\perp Y}$. (The dashed green and blue line identifies a current boundary inferred to occur at the same latitude in both Cluster 3 and Cluster 4). In addition, the currents have been labelled and a downward pointing arrow implies a current flow into the ionosphere and an upward pointing arrow represents current flow out of the ionosphere. Current sheet boundaries can be identified by reversals in the gradient of $\delta B_{\perp Y}$. However, $\delta B_{\perp Y}$ is highly structured with numerous small- and large-scale reversals in the gradient, thereby making identification of the current sheets problematic. In this study only the large-scale, $>20 \mathrm{nT}$ and $>1^{\circ}$, latitude 

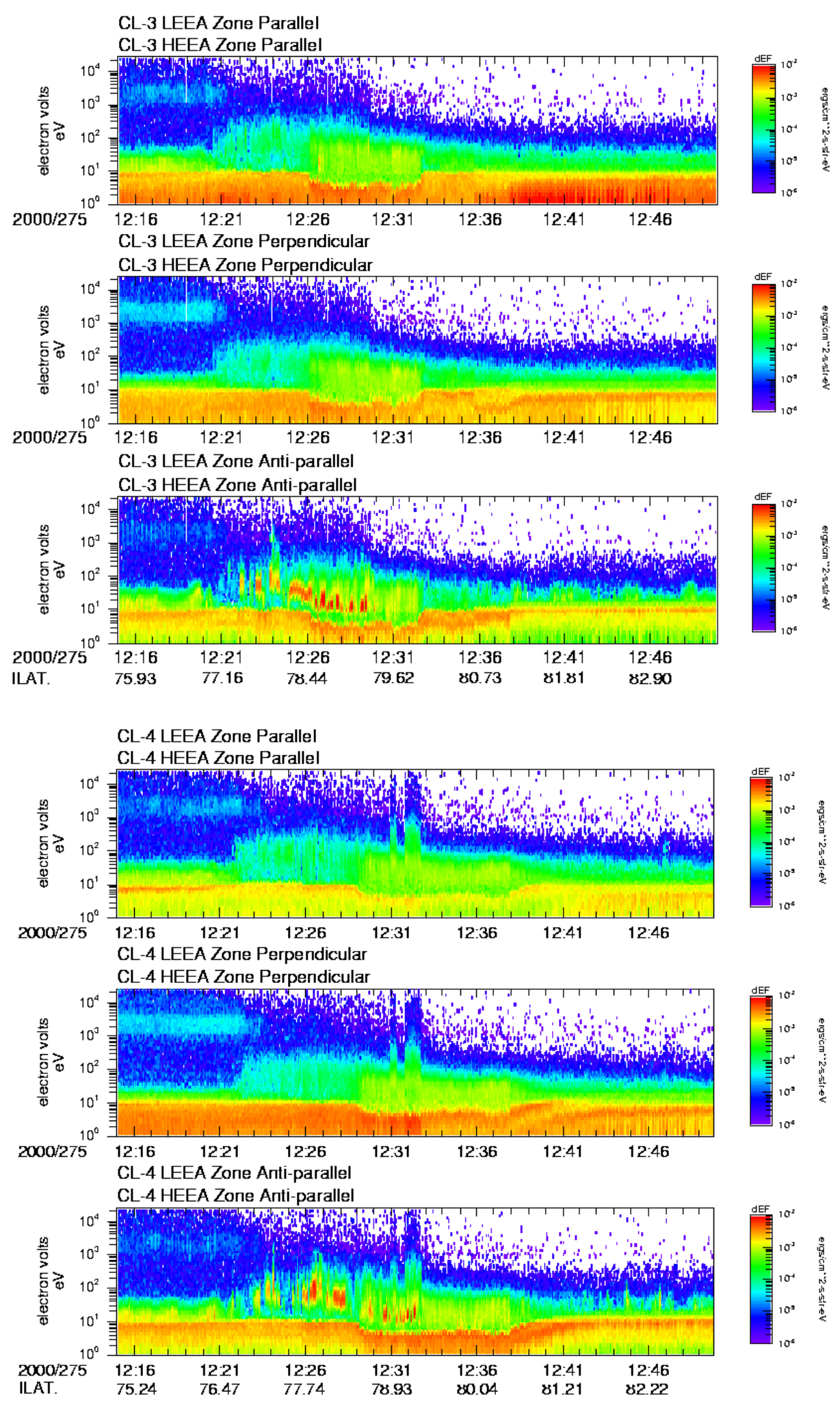

Fig. 2. Electron energy-time spectrograms generated from LEEA and HEEA sensors depicting field-aligned, perpendicular and field opposed electrons, (a) shows Cluster 3 data and (b) shows Cluster 4 data.

changes in the perturbation field are considered. The principal reason for this is that the smaller scale, $\leq 10 \mathrm{nT}$ and $<0.5^{\circ}$, latitude perturbations tend to have similar amplitudes in all three components. Therefore, the assumption that these small-scale perturbations are due to longitudinally extensive current sheets is not valid. 


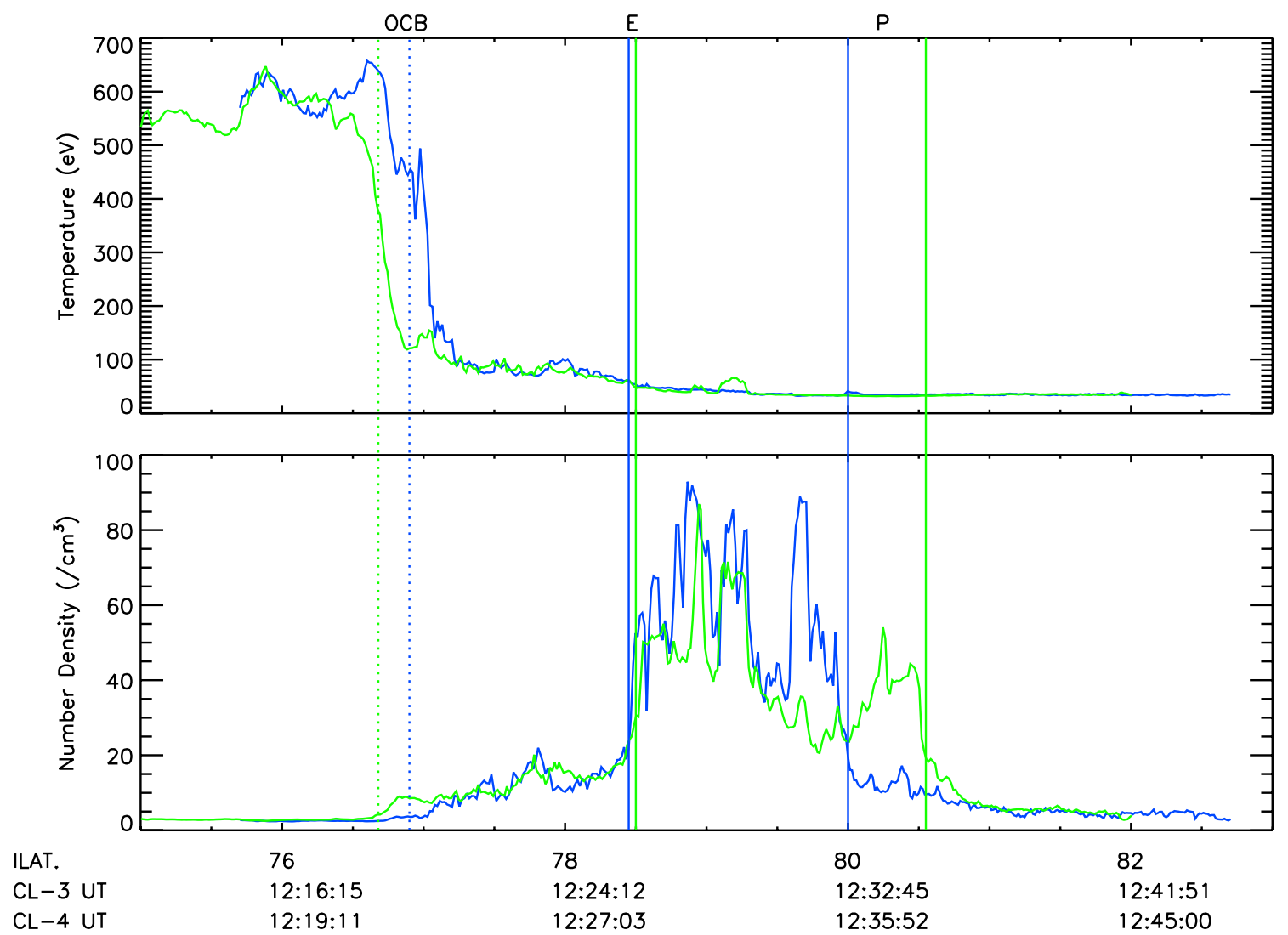

Fig. 3. The upper panel shows the scalar temperature and the lower panel shows the number density moments constructed from HEEA sensor. Blue line refers to Cluster 3 and the green line refers to Cluster 4. The OCB and the equatorward and poleward edges of the cusp are labelled.

Between $79.0^{\circ}$ to $80.0^{\circ}$ latitude in Cluster 3 and $79.0^{\circ}$ to $80.5^{\circ}$ latitude in Cluster 4, there exist small-scale currents. Equatorward of these latitudes, for both data sets, the general trend in the gradient of $\delta B_{\perp Y}$ is positive (with respect to latitude) and is labelled $\mathrm{C} 1$. Poleward of these latitudes, the general trend is negative and is labelled C2. The $\delta B_{\perp Y}$ component amplitude of $\mathrm{C} 1$ and $\mathrm{C} 2$ exceeds the perturbation field amplitude of the other two components; therefore, $\mathrm{C} 1$ and $\mathrm{C} 2$ are considered current sheets. Turning to $\mathrm{C} 2$, the poleward boundary of this current sheet is well-defined, occurring around $82.0^{\circ}$ latitude for both data sets, and identified by the most poleward blue and green vertical lines. The equatorward boundary cannot be defined due to the smallscale currents mentioned above, since $\mathrm{C} 2$ and these smallscale currents may be superposed. Arbitrarily, the equatorward boundary of $\mathrm{C} 2$ is defined by the first small-scale reversal in $\delta B_{\perp Y}$. This results in the equatorward boundary of $\mathrm{C} 2$ being approximately coincident with the poleward edge of the cusp (see bottom panel of Fig. 5) for both data sets (identified by the blue and green vertical lines). Turning to $\mathrm{C} 1$, a similar problem regarding the definition of the poleward boundary of $\mathrm{C} 1$ exists. For consistency with the definition of the equatorward boundary of $\mathrm{C} 2$, the poleward boundary of $\mathrm{C} 1$ is taken at the first small-scale reversal in $\delta B_{\perp Y}$; this occurred at the same latitude for both data sets and is identified by the dashed blue-green line. The equatorward boundary of $\mathrm{C} 1$ in Cluster 4 is identified by a clear reversal in $\delta B_{\perp Y}$ near $77.5^{\circ}$ (identified by the green vertical line). The equatorward boundary of $\mathrm{C} 1$ in Cluster 3 is less easily defined due to the existence of a small-scale current between $77.5^{\circ}$ and $78.0^{\circ}$. Again, the boundary is defined by the first small-scale reversal in $\delta B_{\perp Y}$ which occurs near $78.0^{\circ}$ (identified by the blue vertical line).

Equatorward of $\mathrm{C} 1$, the identification of the current sheets becomes less certain. Between $74.0^{\circ}$ and $77.5^{\circ}$ in Cluster 4 , the general trend in $\delta B_{\perp Y}$ is negative, though there are noticeable small variations in the magnitude of $\delta B_{\perp Y}$. In this latitude range, $\delta B_{\perp Y}$ is significantly larger than the other two components, indicating that this is a longitudinally extended current sheet, which crosses the OCB (vertical dashed green line) with the current boundary $0.9^{\circ}$ poleward of the OCB. Recall that the OCB could not be accurately identified due to the gradual change in the temperature of the electron population. However, it can be seen in Fig. 3 that this poleward boundary is significantly poleward of the temperature transition, indicating that this boundary is on open field lines. Cowley (2000) notes that the Region 1 current flows along the OCB are consistent with the location of this current which is labelled R1.

Turning to Cluster 3, although there is a trend in $\delta B_{\perp Y}$ 

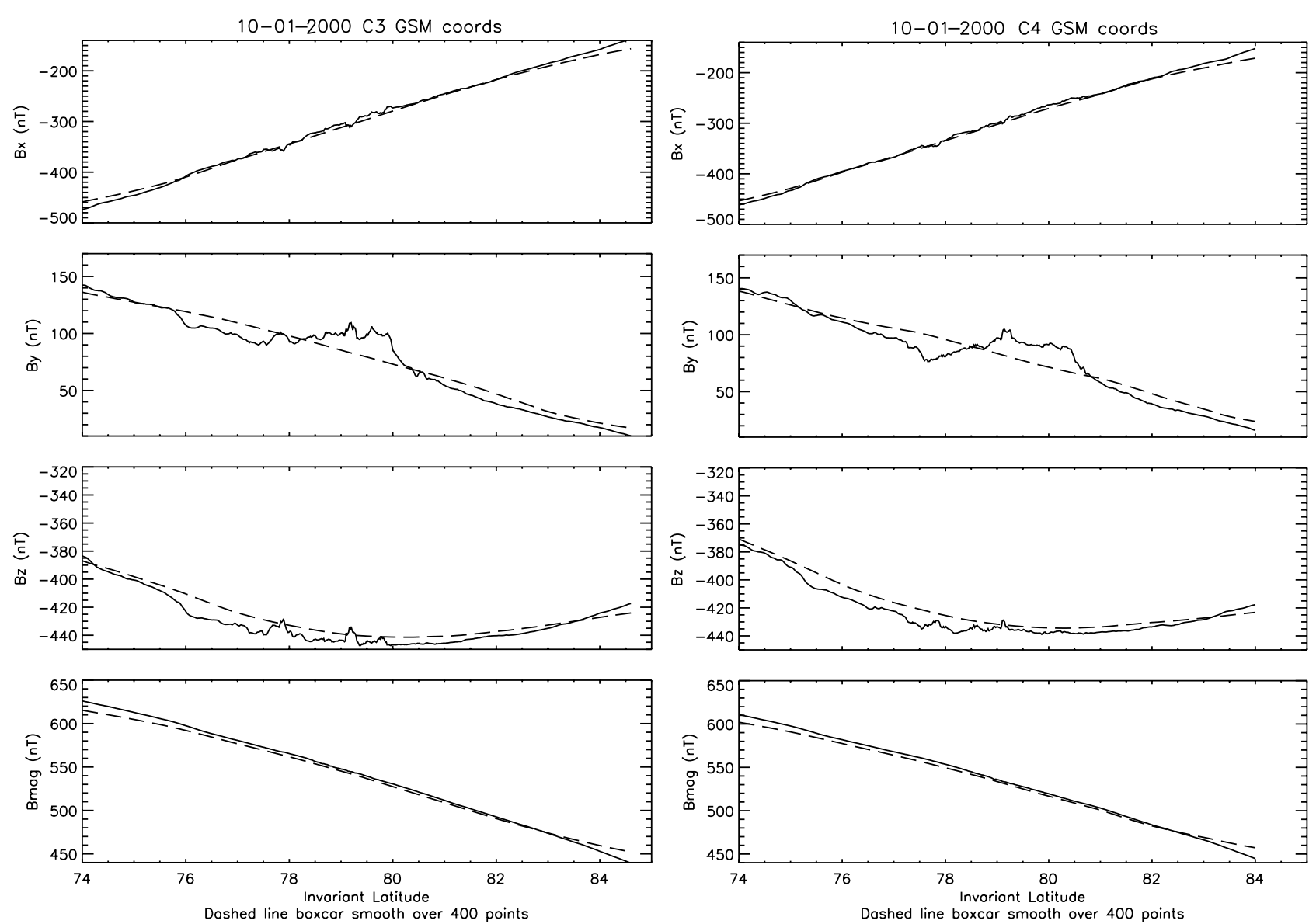

Fig. 4. The three components and magnitude of the total magnetic field (solid line) in GSM coordinates and the background magnetic field (dashed line), (a) from Cluster 3 and (b) from Cluster 4.

between $74.0^{\circ}$ and $77.5^{\circ}$ to more negative values, it is somewhat structured and discontinuous. In addition, there is a significant perturbation field in the other two components. Hence, it is not possible to unambiguously infer the existence of a longitudinally extensive current sheet at Cluster 3 .

Finally, a current sheet is observed poleward of $\mathrm{C} 2$. This is labelled ' $M$ ' since it is in the mantle region.

Since electrons are generally expected to be the principal current carriers in the magnetosphere, an attempt was made to identify those current carriers from the electron spectrograms that are responsible for the field-aligned current sheets inferred from the magnetic field data. Anisotropy between the field-aligned and opposed electron populations can indicate current carriers. These anisotropy can be qualitatively assessed by comparing the top and bottom panels in Figs. 2a and $2 \mathrm{~b}$. The preliminary calibrations used in generating these spectrograms precludes a quantitative calculation. Considering first the higher energy ( $>1 \mathrm{keV}$ ) population, both Cluster 3 and 4 observed an anisotropy in the electron population with an evident excess of field-aligned over opposed electrons. Since Cluster is in the northern hemisphere this implies an excess of electrons moving towards the ionosphere corresponding to an upward current. This is generally con- sistent with the direction of current flow in current sheet R1 inferred from the magnetic field data in Cluster 4 (Fig. 5), though no longitudinally extensive current sheet could be unambiguously inferred from Cluster 3 during this interval. It appears that the anisotropy curtails prior to the inferred current boundary in Cluster 3 and 4. Between 12:22 to 12:30 UT in Cluster 3 and 12:23 to 12:38 UT in Cluster 4, there is an excess of opposed moving electrons. Again, this is generally consistent with the inferred current flow in current sheet $\mathrm{C} 1$ (although placing the equatorward boundary of $\mathrm{C} 1$ in Cluster 3 on the equatorward side of the small-scale current observed in $\delta B_{\perp Y}$ between $77.5^{\circ}$ and $78.0^{\circ}$ would improve the qualitative comparison). The $\mathrm{C} 2$ current sheet occurs immediately poleward of the cusp, roughly coincident with an increase in the energy of the photoelectrons that occurred at 12:32:30 UT in Cluster 3 and at 12:38 UT in Cluster 4. There is some difference in the aligned and opposed electron populations between 100 to $200 \mathrm{eV}$ (green colouring), which possibly indicates an excess of aligned electrons. This is a somewhat tentative interpretation, though this anisotropy is consistent with the current flow in current sheet $\mathrm{C} 2$. However, this anisotropy is inconsistent with the inferred current flow in current sheet $M$. 


\section{Discussion}

The two-point observations of the boundaries deduced from the moment data (Fig. 3, OCB, $E$ and $P$ ) enable the motion of these regions between Cluster 3 and 4 observations to be seen. A schematic illustrating the boundary motion is shown in Fig. 6. Between the Cluster 3 and 4 encounter, the OCB is observed to move equatorward. Two possible interpretations of this motion can be made. One explanation requires a southward turning of the IMF. Recall that the approximate time the IMF turned southward at the magnetopause is 12:28 UT. However, it was demonstrated that there is a significant uncertainty in this timing; hence, it is possible that the observations of the OCB motion were made just after the IMF turned southward. A southward turning of the IMF can cause reconnection near the sub-solar magnetopause which results in a transitory earthward motion of the OCB at the equator. By mapping this motion to the cusp region, we expect a corresponding motion of this boundary (erosion) to lower invariant latitudes which is consistent with the observations. Reconnection in the tail results in a return sunward motion of field lines, thereby restoring flux to the dayside magnetosphere (Smith and Lockwood, 1996). An alternate explanation requires a reduction in the solar wind dynamic pressure. Such a reduction would cause the standoff distance of the sub-solar magnetopause to move sunward. The magnetic fields in the cusp region that close on the dayside would similarly tilt sunward, producing the equatorward motion observed by Cluster 3 and 4. ACE observed a 15\% reduction in the solar wind dynamic pressure between 11:30 to 11:52 UT.

The cusp region was identified by approximately a factor 4 increase in the number density (Fig. 3). Variations in the magnetosheath density near the reconnection site could cause a change in the density of the injected magnetosheath population in the cusp. The distance of ACE from the Earth $\left(\sim 230 R_{E}\right)$ and the uncertainty in the travel time from the ACE to the magnetopause precludes a detailed comparison of the density in the solar wind with the observations made at Cluster. However, between 11:30 to 11:55 UT, the number density observed at ACE was reduced by approximately $15 \%$. This variation is considerable smaller than the observed factor of 4 variation between the cusp and the LLBL/mantle regions. Hence, density variations in the solar wind are unlikely to influence the inferred location of the cusp region, which appears some distance poleward of the OCB since sub-solar reconnected field lines convect poleward in the time taken for injected magnetosheath plasma to reach the mid-altitude cusp region (charge neutrality constrains the electrons to move with the ions; Wing et al., 1996). The equatorward edge of the cusp region did not change in invariant latitude. The authors are aware of no physical reason for this to occur. The most obvious explanation is that the distance the reconnected field line convects during the time taken for the electrons injected at the reconnection site to reach Cluster, combined with the changes in the location of the reconnection site, happen to result in the

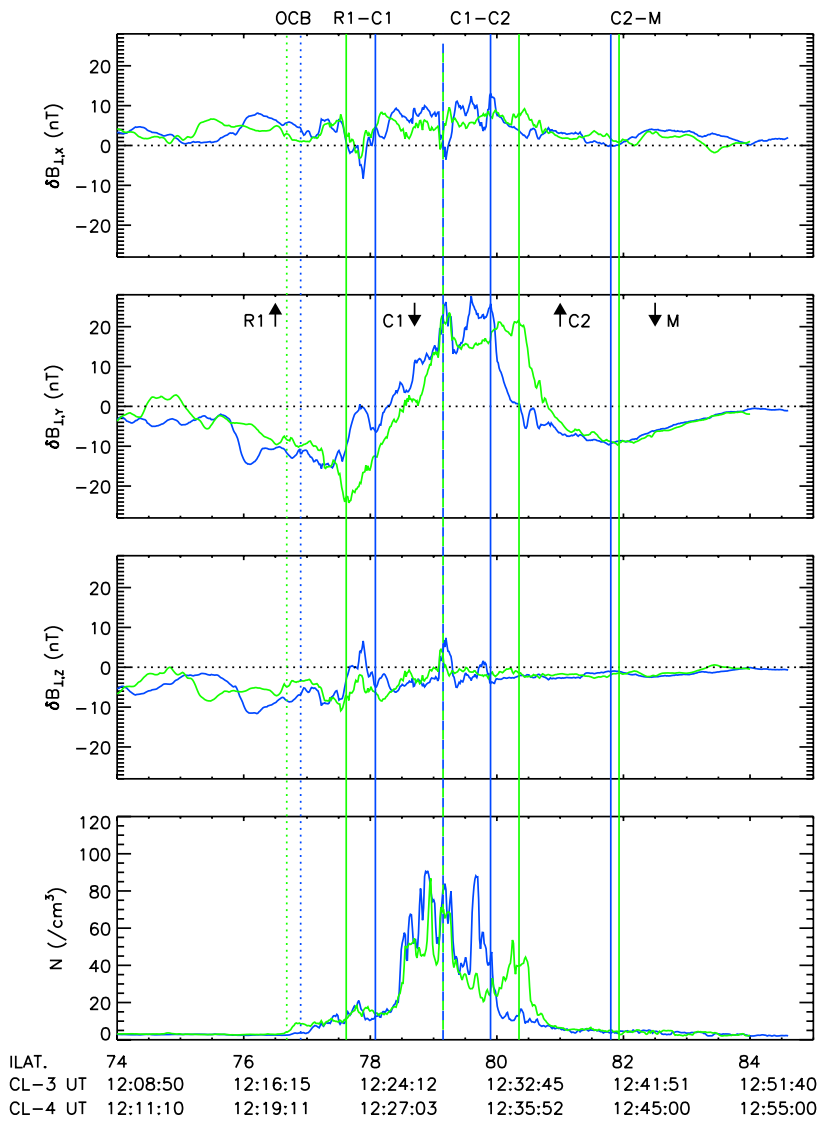

Fig. 5. The three components (GSM) of the perpendicular perturbation magnetic field and electron number density observed from Cluster 3 (blue lines) and Cluster 4 (green lines). The vertical dotted lines identifies the OCB, the vertical green and blue lines identify the current sheet boundaries. The arrows denote the direction of current flow (see text for details).

equatorward edge of the cusp staying at the same latitude. The separation in invariant latitude between the equatorward edge of the cusp, $E$, and the OCB increases by approximately $0.3^{\circ}$ between the encounter of Cluster 3 and the encounter of Cluster 4 (Fig. 3). This could be explained by an increase in the convection velocity of the reconnected field lines resulting in these field lines moving further poleward before the injected magnetosheath plasma reaches the Cluster spacecraft. Alternatively, the distance to the reconnection site could increase and so the time taken for the injected magnetosheath plasma to reach the Cluster spacecraft increases, allowing for the reconnected field lines to convect further poleward. Finally, the flaring angle (the difference in invariant latitude between $P$ and $E$ shown in Fig. 3) of the cusp region increases by approximately $0.6^{\circ}$.

Observations of the magnetic field indicated that Cluster passed through four longitudinally extended field-aligned current sheets (though there is some uncertainty as to whether Cluster 3 observed the Region 1 current sheet). de la Beaujardiere et al. (1993) used a similar technique to study the Birkeland current system, and observed the Region 1 and 


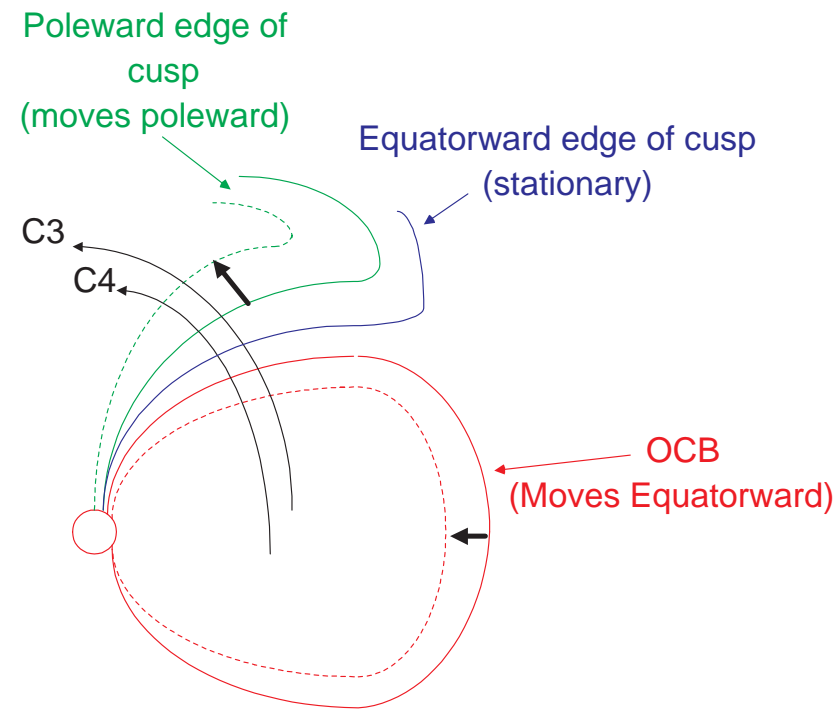

Fig. 6. Diagram depicting the motion of the boundaries identified in Fig. 3. The solid lines depict Cluster 3 and the dashed lines depict Cluster 4. Red denotes the OCB, blue denotes the equatorward edge of the cusp (since this is stationary, there is no dashed line) and green denotes the poleward edge of the cusp.

Region 2 current sheets and one other current sheet poleward of the Region 1 current sheet. Their observations were consistent with Cowley et al. (1991). The most equatorial current sheet we have observed in the previous section straddled the OCB and is taken to be the Region 1 current sheet (Cowley et al., 1991). Since the Region 1 current sheet is expected to lie poleward of the Region 2 current sheet (Cowley, 2000), then we observe two additional current sheets in comparison to de la Beaujardiere et al. (1993). Recall that ACE observed a southward turning of the IMF at 11:33 UT. Based on the observed solar wind velocity, this turning would arrive at the magnetopause at approximately 12:28 UT. Hence, the observations made during this pass could be coincident with a reorganization of the polar convection pattern. This could be a possible explanation for the greater number of current sheets observed here. However, significant uncertainty in the precise travel time from ACE to the magnetopause exists; hence, it is not possible to state unambiguously that these observations are a result of the reorganisation of the largescale convection in the polar magnetosphere. Moreover, an assessment of such a reorganization would require more than a single case study presented here and is therefore beyond the scope of this paper. The boundary between the Region 1 and $\mathrm{C} 1$ current sheets, identified by the solid and dotted lines observed by Cluster 3 at $76.4^{\circ}$ and by Cluster 4 at $76.6^{\circ}$ (Fig. 5), occurred within the LLBL region. This current boundary moved equatorward along with the OCB. However, a smallscale current pair observed by Cluster 3 around $77.8^{\circ}$ latitude may effect the identification of the equatorward boundary of $\mathrm{C} 1$ in Cluster 3. Recall that the equatorward boundary of $\mathrm{C} 1$ in Cluster 3 would be, qualitatively, more consistent with the current inferred from the anisotropy between the aligned and opposed electron populations if this boundary was placed around $77.5^{\circ}$. In this case, the motion of the OCB would not be in accord with the motion of the equatorward boundary of $\mathrm{C} 1$. The current sheet marked $\mathrm{C} 1$ in Fig. 5 straddled a significant fraction of the LLBL and the cusp regions. Small-scale currents observed between $79^{\circ}$ and $80^{\circ}$ latitude in Cluster 3 and $79^{\circ}$ and $80.5^{\circ}$ in Cluster 4 were used to define the poleward limit of $\mathrm{C} 1$. Using this criteria effectively, served to define a minimum poleward extent of C1. Cowley (2000) proposes that a cusp current would flow on the equatorial edge of the cusp (The direction of the current flow is dependent on the hemisphere, the MLT, and the IMF $B_{Y}$ component). Observations made here indicate that the current sheet is by no means confined to the equatorial edge of the cusp. There is a striking contrast between current and precipitation boundaries. One possible explanation of this difference is the 'morphological' description of the precipitation regions. Fuselier et al. (1999) suggest that both the LLBL and cusp regions (when the IMF is southward) contain reconnected field lines. The difference in the characteristics of the plasma in the LLBL and cusp regions is attributed to the differences in the characteristics of the source population in the magnetosheath. Hence, the equatorward edge of the cusp does not denote the first observation of magnetosheath plasma. In addition, Lockwood et al. (1998) propose a variation in the reconnection rate, including completely pulsed reconnection, $E_{T}=0$. Variation in the reconnection rate implies variation in the stress at the magnetopause. This, in turn, would influence the current flow, resulting in a more complex picture than the current flowing at the edge of the cusp.

Cowley (2000) also states that a current sheet should flow along the poleward edge of the cusp. The definition used to define the equatorward boundary of $\mathrm{C} 2$ results in this boundary being coincident with the poleward edge of the cusp. It can be readily seen in Fig. 5 that there is a noticeable steepening in the magnitude of the gradient of $\delta B_{\perp Y}$ close to the poleward edge of the cusp, suggesting that the definition of the equatorward boundary of $\mathrm{C} 2$ is reasonable. In addition, given this definition, both the poleward edge of the cusp and the equatorward boundary of $\mathrm{C} 2$ move poleward by a similar amount.

The most poleward current sheet observed in Fig. 5, marked $M$, occurs in the mantle region and does not change significantly between the encounter of Cluster 3 and the encounter of Cluster 4 of this current. Recall that the background field, used in calculating the perturbation field, was generated by smoothing the total field with a 400 point running mean boxcar filter. It is possible that this process could introduce field perturbation. In an attempt to validate the perturbation field, several background fields were calculated using boxcar filters ranging from 100 to 800 sample points. The perturbation fields generated by subtracting these background fields from the total field were all qualitatively similar, i.e. 4 current sheets could be inferred from the perturbation field. This indicates that the current sheet $M$ is probably not a facet of the procedure used to calculate $\delta B_{\perp}$.

Finally, an attempt was made to qualitatively relate the 
currents inferred from the field data to anisotropy between the aligned and opposed electron populations. In general, there was some agreement between these electron anisotropy and the inferred direction of current flow in $\mathrm{R} 1, \mathrm{C} 1$ and $\mathrm{C} 2$ (though there was some uncertainty over whether Cluster 3 observed the Region 1 current sheet). However, the direction of the current flow inferred from the anisotropy was found to be inconsistent with current sheet $M$. Obviously, a quantitative comparison, which is beyond the scope of this paper, would have to be performed in order to resolve this inconsistency.

\section{Conclusions}

The dynamic nature of the cusp region has been demonstrated by this preliminary two-point study. Observations of the OCB were consistent with ongoing sub-solar reconnection which is probably expected to occur based on ACE data. The change in the separation in invariant latitude between the equatorward edge of the cusp region and the OCB indicated a possible change in convection velocity and/or a change in the location of the reconnection site. Four longitudinally extended field-aligned current sheets were inferred from the magnetic field data. This number of current sheets may have resulted from a reorganization of the polar convection pattern driven by a southward turning of the IMF. Two of these current sheets were related to the equatorward and poleward edges of the cusp region. Cluster will return to the mid-altitude cusp one year after these observations were made, thus enabling a four-point study of this region to be made.

Acknowledgements. The authors would like to thank M. Lockwood for the useful discussions, N. Ness for use of the ACE/MFI data, D. J. McComas for use of the ACE/SWE data. We acknowledge CDAWeb for access to ACE data. This work was funded by PPARC.

Topical Editor G. Chanteur thanks two referees for their help in evaluating this paper.

\section{References}

André, M., Crew, G. B., Peterson, W. K., Persoon, A. M., Pollock, C. J., and Engebretson, M. J.: Ion heating by broadband lowfrequency waves in the cusp/cleft, J. Geophys. Res., 95, 20 809$20823,1990$.

Balogh, A., Carr, C. M., Acuña, M. H., et al.: The Cluster magnetic field investigation: overview of in-flight performance and initial results, Ann. Geophysicae, this issue, 2001.

Cowley, S. W. H, Morelli, J. P., and Lockwood, M.: Dependence of convective flows and particle precipitation in the high-latitude dayside ionosphere on the $X$ and $Y$ components of the interplanetary magnetic field, J. Geophys. Res., 96, 5557-5564, 1991.

Cowley, S. W. H.: Magnetosphere-Ionosphere interactions: A tutorial review, in Magnetospheric Current Systems, Geophys. Monogr. Ser., (Eds) Ohtani, S.-I., Fujii, R., Hesse, M., and Lysak, R. L., 118, 91-106, AGU, Washington, D.C., 2000.

de la Beaujardiere, O., Waterman, J., Newell, P., and Rich, F.: Relationship between Birkeland current regions, particle precipitation and electric fields, J. Geophys. Res., 98, 7711-7720, 1993.
Dungey, J. W.: Interplanetary magnetic field and the auroral zones, Phys. Rev. Lett., 6, 47, 1961.

Erlandson, R. E., Zanetti, L. J., Potemra, T. A., Bythrow, P. F., and Lundin, R.: IMF $B_{Y}$ dependence of Region 1 Birkeland currents near noon, J. Geophys. Res., 93, 9804-9814, 1988.

Franz, J. R., Kintner, P. M., and Pickett, J. S.: Polar observations of coherent electric field structures, Geophys. Res. Lett., 25, 12771280, 1998.

Fuselier, S. A., Lockwood, M., Onsager, T. G., and Peterson, W. K.: The source population for the cusp and cleft/llbl for southward IMF, Geophys. Res. Lett., 26, 1665-1668, 1999.

Hones, Jr., E. W.: (Ed) Magnetic Reconnection in Space and Laboratory Plasmas, Geophys. Monogr. Ser., Vol. 30, AGU, Washington, D.C., 1984.

Hultqvist, B., Vo, H., Lundin, R., Aparicio, B., Lindqvist, P.-A., Gustafsson, G., and Holback, B.: On the upward acceleration of electrons and ions by low-frequency electric field fluctuations observed by Viking, J. Geophys. Res., 96, 11 609-11 615, 1991.

Iijima, T. and Potemra, T. A.: Field-aligned currents in the dayside cusp observed by Triad, J. Geophys. Res., 81, 5971-5979, 1976.

Johnstone A. D., Alsop, C., Burge, S., Carter, P. J., Coates, A. J., Coker, A. J., Fazakerley, A. N., Grande, M., Gowen, R. A., Gurgiolo, C., Hancock, B. K., Narheim, B., Preece, A., Sheather, P. H., Winningham, J. D., and Woodliffe, R. D.: 'Peace: A Plasma electron and current experiment', Space Sci. Rev., 79, 351-398, 1997.

Lockwood, M. and Smith, M. F.: Low and middle altitude cusp particle signatures for general magnetopause reconnection rate variations: 1. Theory, J. Geophys. Res., 99, 8531-8553, 1994.

Lockwood, M. and Hapgood, M. A.: On the cause of a magnetospheric flux transfer event, J. Geophys. Res., 103, 26 453-26478, 1998.

Lockwood, M., Davis, C. J., Onsagar, T. G., and Scudder, J. D.: Modelling signatures of pulsed reconnection in cusp ion dispersion signatures seen at middle altitudes, Geophys. Res. Lett., 25, 591-594, 1998.

Newell, P. T. and Meng, C.-I.: The cusp and the cleft/boundary layer: Low-altitude identification and statistical local time variation, J. Geophys. Res., 93, 14 549-14 556, 1988.

Reiff, P. H., Burch, J. L., and Spiro, R. W.: Cusp proton signatures and the interplanetary magnetic field, J. Geophys. Res., 85, 5997-6005, 1980.

Rosenbauer, H., Grunwaldt, H., Montgomery, M. D., Paschmann, G., and Sckopke, N.: Heos 2 plasma observations in the distant magnetosphere: The plasma mantle, J. Geophys. Res., 80, 27232737, 1975.

Rostoker, G.: Magnetospheric and ionospheric currents in the polar cusp and their dependence on the $B_{Y}$ component of the interplanetary magnetic field, J. Geophys. Res., 85, 4167-4176, 1980.

Russell, C. T. and Elphic, C.: ISEE observations of flux transfer events at the dayside magnetopause, Geophys. Res. Lett., 6, 3336, 1979.

Smith, M. F. and Lockwood, M.: Earth's magnetospheric cusps, Rev. Geophy. 34, 233-260, 1996.

Wing, S., Newell, P. T., and Onsager, T. G.: Modelling the entry of magnetosheath electrons into the dayside ionosphere, J. Geophys. Res., 101, 13 155-13 167, 1996.

Woch, J. and Lundin, R.: Magnetosheath plasma precipitation in the polar cusp and its control by the inter-planetary magnetic field, J. Geophys. Res., 97, 1421-1430, 1992. 